\title{
Modelo ontosemiótico de referencia de la proporcionalidad: Implicaciones para la planificación curricular en primaria y secundaria
}

\author{
María Burgos, Universidad de Granada. mariaburgos@ugr.es \\ Juan D. Godino, Universidad de Granada.jgodino@ugr.es
}

\begin{abstract}
Modelo ontosemiótico de referencia de la proporcionalidad: Implicaciones para la planificación curricular en primaria y secundaria

\section{Resumen}

El diseño curricular de un contenido matemático en los diversos niveles educativos requiere considerar la diversidad de significados y su articulación progresiva, atendiendo a los grados de generalidad y formalización. En este trabajo se analizan los diversos significados de la proporcionalidad aplicando herramientas teóricas del Enfoque Ontosemiótico. En particular, tratamos la interpretación del significado en términos de sistemas de prácticas operativas y discursivas relativas a la resolución de tipos de problemas y el modelo de niveles de algebrización de la actividad matemática. De acuerdo con investigaciones previas se identifican tipos de situaciones-problemas y configuraciones de prácticas, objetos y procesos que permiten identificar significados parciales (intuitivo, aritmético, protoalgebraico y algebraico) que deben ser tenidos en cuenta en la planificación y gestión de los procesos de enseñanza y aprendizaje de la proporcionalidad en educación primaria y secundaria.
\end{abstract}

Palabras clave. Proporcionalidad; significados pragmáticos; configuración ontosemiótica; diseño curricular; niveles de algebrización.

Ontosemiotic reference model of proportionality: Implications for curricular design in primary and secondary education

\section{Abstract}

The curricular design of a mathematical content at the different educational levels requires considering the diversity of meanings and their progressive articulation, by attending to the degrees of generality and formalization. In this paper, we analyze the different meanings of proportionality, by applying the Ontosemiotic Approach theoretical tools. In particular, we undertake the interpretation of meaning in terms of systems of operative and discursive practices related to the resolution of problem types and the model of mathematical activity algebrization levels. Drawing on prior research, we identify types of situations-problems and configurations of practices, objects and processes, in order to identify partial meanings (intuitive, arithmetic, proto-algebraic and algebraic) that must be taken into account in planning and managing teaching and learning processes of proportionality in primary and secondary education.

Keywords. Proportionality; pragmatic meanings; ontosemiotic configuration; curriculum design; algebrization levels.

\section{Introducción}

El estudio de las razones, proporciones y proporcionalidad es un tema importante en el currículo escolar que se inicia en educación primaria y continúa en secundaria, siendo transversal a diferentes materias (Wilhelmi, 2017). Esta presencia longitudinal y transversal de la proporcionalidad justifica la gran cantidad de investigaciones que se han realizado sobre los problemas que plantea su enseñanza y aprendizaje (Cramer \& Post, 1993; Lamon, 2007, 2012; Obando, Vasco \& Arboleda, 2014; Tourniaire \& Pulos, 1985). 
Desde el enfoque cognitivo de investigación en didáctica de la matemática son múltiples las investigaciones que han abordado el problema de la enseñanza y del aprendizaje de la proporcionalidad. Tournaire y Pulos (1985), Fernández y Llinares (2012) y Silvestre y Ponte (2011), entre otros, han analizado las características del desarrollo del razonamiento proporcional, mostrando las dificultades que encuentran los estudiantes de distintos niveles educativos al afrontar situaciones de proporcionalidad. En particular, los resultados de estos trabajos muestran que el logro en las tareas de proporcionalidad depende de diversos factores: la relación entre los números implicados, el uso de razones enteras y no enteras, las unidades de las magnitudes involucradas en la situación, la manera en que se formula la tarea, la familiaridad con el contenido, etc.

En cuanto a la instrucción, se propone anticipar una aproximación informal al concepto de razón y proporción previa a su formalización y algoritmización, defendiendo una secuencia didáctica que permita avanzar desde un conocimiento de naturaleza intuitiva y cualitativa, de estructura aditiva, hacia uno cuantitativo de estructura multiplicativa (Streefland, 1985; Behr, Harel, Post \& Lesh 1992; Cramer \& Post, 1993).

En cuanto al conocimiento matemático institucionalizado, la proporcionalidad ha sido estudiada desde tres puntos de vista: el aritmético, centrado en la noción de razón y proporción; el algebraico, centrado en la noción de función; y el geométrico, centrado en la noción de semejanza. El enfoque aritmético del estudio de la proporcionalidad predomina en la mayoría de propuestas curriculares e investigaciones (Ben-Chaim, Keret \& Ilany, 2012; Lamon, 2007). Se suele asumir que una razón es una comparación multiplicativa entre cantidades de una magnitud o entre dos magnitudes, que la proporción es una igualdad de dos razones y la proporcionalidad de magnitudes es una función lineal entre las cantidades de dichas magnitudes. Se acepta que estas definiciones captan la esencia de los conceptos y, de ahí, el problema de su enseñanza consiste en hacer que los estudiantes comprendan estas conceptualizaciones y sus aplicaciones.

En el marco de la Teoría Antropológica de lo Didáctico (TAD, Chevallard, 1992), razón, proporción y proporcionalidad se comprenden en términos de organizaciones matemáticas complejas determinadas por tipos de situaciones, prácticas matemáticas, técnicas, tecnologías y teorías, estructuradas alrededor de praxeologías institucionales (Bolea, Bosch \& Gascón, 2001; Bosch, 1994; García, 2005). García (2005) asume el modelo epistemológico sobre la proporcionalidad iniciado en los trabajos de Bolea et al. (2001) y Bolea (2002) a partir del hipotético proceso de algebrización de los sistemas proporcionales, ampliando el campo de estudio a un conjunto de relaciones entre magnitudes, entre las que la relación de proporcionalidad es una relación más.

Comin (2000) propone un modelo en el que organiza los conocimientos de la proporcionalidad en tres marcos: el de las magnitudes, el de las medidas de magnitud y el de las variables numéricas, analizando desde el punto de vista epistémico, los objetos que constituyen el entorno de la proporcionalidad (magnitudes, cantidades, razones, proporciones). Así, asume la función lineal como una abstracción que resume y refleja la relación de proporcionalidad entre magnitudes, mostrando la estrecha relación entre las nociones de variable, función y número.

Aunque la perspectiva epistémica sobre la proporcionalidad en los trabajos de Bosch (1994), Comin (2000) y García (2005) amplía la visión aritmética usualmente implementada en los currículos, diversas teorías didácticas están proponiendo una visión más compleja sobre los significados de cualquier objeto matemático, esto es, una visión epistemológica de las matemáticas más rica (Steinbring, 1997; Gómez, 2007). Tal es el caso del Enfoque ontosemiótico (EOS) del conocimiento y la instrucción matemáticos 
(Godino \& Batanero, 1994; Godino, Batanero \& Font, 2007), que entiende las matemáticas como una actividad de las personas implicadas en la solución de cierta clase de situaciones - problemas, e interpreta el significado institucional y personal de los objetos matemáticos en términos de los sistemas de prácticas operativas y discursivas que se ponen en juego en la solución de dichas situaciones.

En este trabajo se aborda el problema de caracterizar los diversos significados de la proporcionalidad y su articulación en una trama estructurada en base a los diversos grados de generalidad y formalización que se ponen en juego. Como se indica en Godino, Beltrán-Pellicer, Burgos y Giacomone (2017), el universo de significados de la proporcionalidad se puede clasificar según criterios, en particular, el contexto o campo de aplicación y el nivel de algebrización de las prácticas matemáticas realizadas. Algunos contextos de aplicación de las nociones de razón y proporción (vida cotidiana, científicotécnico, artístico, geométrico, probabilístico, estadístico, etc.) conllevan la participación de objetos y procesos específicos de dichos campos en las prácticas de resolución de los problemas correspondientes, como revelan las múltiples investigaciones realizadas sobre la naturaleza y desarrollo del razonamiento proporcional (Freudenthal, 1983; Lamon, 2007; Tourniaire \& Pulos, 1985). En consecuencia, se pueden delimitar variantes de significados propios de algunos campos de aplicación de la proporcionalidad (geométrico, probabilístico, etc.). En este trabajo proponemos un modelo para categorizar los significados de la proporcionalidad según el nivel de algebrización de las prácticas matemáticas, esto es, según el grado de generalidad de los objetos implicados, de los lenguajes usados y del cálculo analítico que se realiza con dichos objetos.

Tras esta introducción se describen problema, marco teórico y método. Los estudios sobre proporcionalidad que hemos mencionado serán tenidos en cuenta en la elaboración de los diversos significados parciales que se describen en la sección 3. En la sección 4 se elabora una síntesis de los significados, estructurados según los niveles de algebrización implicados y su distribución en las etapas educativas, comenzando en educación primaria y terminando en bachillerato y primeros cursos de universidad. Finalmente se incluyen implicaciones del trabajo para el desarrollo curricular y la formación de profesores.

\section{Problema, marco teórico y método}

El análisis global de los procesos de instrucción matemática no se puede realizar descontextualizado por ser consustancial a la institución y al tiempo (Wilhelmi, 2017). Se necesita describir un significado global u holosignificado (Wilhelmi, Godino \& Lacasta, 2007) que oriente el desarrollo del currículo y articule significados parciales adquiridos. En consecuencia, las cuestiones que abordamos en este trabajo sobre la proporcionalidad son las siguientes: i) ¿Qué significados se pueden identificar para la proporcionalidad? ii) ¿Cómo se distinguen tales significados según grado de generalidad y formalización? iii) ¿Cómo se relacionan y articulan entre sí los diversos significados? iv) ¿En qué etapa educativa y de qué forma se puede abordar su estudio?

Para responder a estas cuestiones es necesario adoptar una teoría sobre el significado de los objetos matemáticos que asuma como postulado su relatividad institucional y contextual y que permita, además, indagar las características de los mismos. En nuestro caso consideramos que la noción de significado institucional que propone el EOS, entendido en términos pragmáticos como "sistemas de prácticas operativas y discursivas" permite abordar las cuestiones mencionadas de manera eficaz.

El presente estudio de los significados de la proporcionalidad amplía Godino et al. (2017) y complementa otros trabajos en el marco del EOS sobre caracterización de 
significados institucionales (Batanero, 2005; Wilhelmi et al., 2007; Godino, Font, Wilhelmi \& Lurduy, 2011; Pino-Fan, Godino \& Font, 2011), al proponer una articulación entre los diversos significados según nivel de algebrización de las prácticas matemáticas.

\subsection{Significado pragmático y configuración ontosemiótica}

Dos nociones clave del EOS son las de significado y configuración ontosemiótica de prácticas, objetos y procesos. Se considera práctica matemática a "toda actuación o expresión (verbal, gráfica, etc.) realizada por alguien para resolver problemas matemáticos, comunicar a otros la solución obtenida, validarla o generalizarla a distintos contextos y problemas" (Godino \& Batanero, 1994, p. 334). Dado que un objeto matemático, en su versión institucional se concibe como un "emergente del sistema de prácticas sociales asociadas a un campo de problemas" (p. 335), el significado de un objeto queda determinado por el "sistema de prácticas institucionales asociadas al campo de problemas de las que emerge el objeto en un momento dado" (p. 338).

Identificado un significado para un objeto matemático, se tiene un tipo de situaciónproblema, que se puede concretar en un ejemplar prototípico y la secuencia de prácticas necesarias para resolverlo. El reconocimiento de la trama de objetos interrelacionados que interviene en dichas prácticas es necesario para gestionar los procesos de estudio matemáticos y tomar conciencia de la complejidad ontosemiótica de la actividad matemática como un factor explicativo de las dificultades en dichos procesos.

Para realizar el análisis de las prácticas matemáticas, el EOS ha introducido la noción de configuración ontosemiótica de prácticas, objetos y procesos, en la que los tipos de objetos según su naturaleza y función son clasificados en las siguientes categorías:

- Lenguajes (términos, expresiones, notaciones, gráficos) en sus diversos registros (escrito, oral, gestual, etc.).

- Situaciones-problemas (aplicaciones extra-matemáticas, ejercicios).

- Conceptos (introducidos mediante definiciones o descripciones).

- Proposiciones (enunciados sobre conceptos).

- Procedimientos (algoritmos, operaciones, técnicas de cálculo).

- Argumentos (enunciados para justificar las proposiciones y procedimientos deductivos o de otro tipo).

Los objetos matemáticos que intervienen y emergen de los sistemas de prácticas matemáticas se relacionan entre sí. Así, las situaciones-problemas son la razón de ser de la actividad matemática; el lenguaje constituye el instrumento de trabajo matemático y representa las demás entidades; los argumentos fundamentan los procedimientos y las proposiciones que relacionan los conceptos matemáticos entre sí.

\subsection{Niveles de algebrización}

En Godino, Aké, Gonzato y Wilhelmi (2014) se propone un modelo de razonamiento algebraico para la Educación Primaria basado en la distinción de tres niveles de algebrización. Los criterios para delimitar los niveles están basados en el tipo de objetos y procesos matemáticos implicados en la actividad matemática: representaciones usadas, procesos de generalización implicados (de los que emergen objetos generales o intensivos, en estrecha relación con los objetos particulares o extensivos) y cálculo analítico que se pone en juego en la actividad matemática correspondiente:

- Nivel 0. Se opera con objetos intensivos de primer grado de generalidad, usando lenguajes natural, numérico, icónico, gestual. 
- Nivel 1. Involucra objetos intensivos de segundo grado de generalidad, propiedades de la estructura algebraica de $\mathrm{N}$ y la igualdad como equivalencia.

- Nivel 2. Se usan representaciones simbólico- literales para referir a objetos intensivos reconocidos ligados a la información espacial, temporal y contextual; se resuelven ecuaciones de la forma $\mathrm{A} x+\mathrm{B}=\mathrm{C}$.

- Nivel 3. Los símbolos se emplean de manera analítica, sin referir a la información contextual. Se realizan operaciones con indeterminadas o variables; se resuelven ecuaciones de la forma $\mathrm{A} x+B=\mathrm{C} x+\mathrm{D}$.

En Godino, Neto, Wilhelmi, Aké, Etchegaray y Lasa (2015) se extiende el modelo de algebrización a la actividad matemática propia de Educación Secundaria y Bachillerato, describiendo tres niveles adicionales para esta etapa. El uso y tratamiento de parámetros es un criterio para definir niveles superiores de algebrización, al estar vinculado a la presencia de familias de ecuaciones y funciones, y por tanto, implica nuevos niveles de generalidad. El lenguaje empleado en estos niveles es simbólico-literal; los símbolos se usan de forma analítica, sin referir a información contextual.

- Nivel 4. Primer encuentro con parámetros para expresar familias de ecuaciones y funciones. Se opera con coeficientes variables pero no con parámetros.

- Nivel 5. Tratamiento de parámetros. Se realizan cálculos analíticos (sintácticos) en los que intervienen uno o más parámetros, conjuntamente con otras variables.

- Nivel 6. Introducción de algunas estructuras algebraicas (como la de espacio vectorial, o grupo) y el estudio del álgebra de funciones.

\subsection{Método}

En Godino (2002) se desarrolla una técnica para el análisis ontosemiótico de los significados puestos en juego en un proceso de instrucción implementado, o planificado en una lección de un libro de texto. Se procede a identificar la trama de objetos y funciones semióticas que se establecen en las prácticas operativas y discursivas elementales correspondientes. En este caso se trata de un análisis con un fuerte carácter microscópico.

Para la caracterización de los significados institucionales que sirvan de referencia global para el diseño curricular es necesario adoptar un punto de vista macroscópico, teniendo en cuenta las investigaciones sobre el tema. En este análisis ontosemiótico se comienza con la selección de las situaciones-problemas y distintas maneras de abordar su resolución, en las cuales interviene el objeto bajo estudio de manera crítica. En las prácticas operativas y discursivas que se deben realizar para resolver tales problemas intervienen, además, otros objetos lingüísticos, conceptuales, procedimentales, proposicionales y argumentativos que ponen en juego diferentes grados de generalidad y formalización, permitiendo definir significados parciales del objeto y establecer relaciones jerárquicas entre ellos en función de su complejidad e interdependencia.

\section{Análisis y resultados: Significados parciales de la proporcionalidad}

En Godino et al. (2017) se distinguen tres tipos de significados de la proporcionalidad: aritmético, protoalgebraico y algebraico-funcional, que además se complementan con un significado informal-cualitativo, a partir de la aplicación de los niveles de algebrización (sección 2.2.) a los sistemas de prácticas ligados a tareas relativas a proporcionalidad. En esta sección ampliamos dicho modelo conectando los niveles descritos con los propios de niveles educativos superiores. Para cada significado proponemos situaciones-problemas características e identificamos los objetos implicados en la resolución. 


\subsection{Significado intuitivo-cualitativo}

Un razonamiento de tipo intuitivo, basado en la comparación perceptiva para determinar la semejanza de figuras o formas planas, supone un primer acercamiento a las relaciones de proporcionalidad en el contexto geométrico (Fiol \& Fortuny, 1990). Un ejemplo de tarea (Figura 1) conduce a los estudiantes a reconocer perceptivamente las relaciones proporcionales entre formas de figuras dibujadas a escala sin considerar las relaciones de tipo cuantitativo entre las razones de segmentos (Ruiz \& Lupiáñez, 2009).

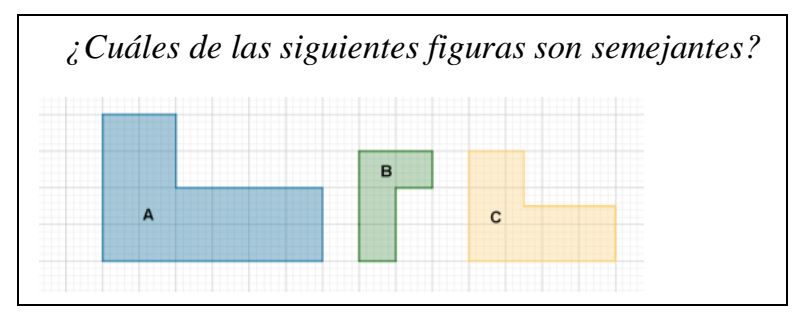

Figura 1. Ejemplo de tarea de semejanza de figuras

Argumentos de tipo cualitativo, que recaen en la comparación o análisis de las relaciones multiplicativas entre números particulares, son el primer paso para distinguir si estamos ante una situación de proporcionalidad y si ésta es directa o inversa. Los tipos de problema abordables con razonamiento pre-proporcional e informal son los que involucran comparación de razones (Behr et al., 1992). Este tipo de significado es el que se moviliza en un problema como el siguiente (adaptado de Godino et al., 2017, p. 5):

Si Juan mezcla menos concentrado de limón con más azúcar que la que la que prepara su amiga María, su limonada tendrá un gusto: (a) Más fuerte; (b) Menos fuerte; (c) Exactamente el mismo gusto.

El carácter informal del argumento refiere a admitir que existe una relación entre las magnitudes consideradas de la forma "más concentrado de limón, más azúcar". El significado intuitivo-cualitativo se apoya sobre el conocimiento y sentido de las operaciones y la experiencia del individuo, suponiendo un conocimiento de la proporcionalidad no institucionalizado como saber que le lleve a argumentar que menos concentrado de limón con más cantidad de azúcar proporcionará un saber menos fuerte.

\subsection{Significado aritmético}

El significado aritmético se caracteriza por la aplicación de procedimientos de cálculo aritméticos (multiplicación, división). Este tipo de significado aparece en el siguiente problema adaptado de Lamon (2007, p. 637).

Problema 1. Juan prepara una limonada utilizando 3 cucharadas de azúcar y 12 cucharadas de concentrado de jugo de limón. Mientras María utiliza 5 cucharadas de azúcar y 20 cucharadas de concentrado de jugo de limón. ¿Cuál de las dos limonadas es más dulce, la de Juan o la de María? ¿O tienen el mismo gusto?

En una solución de tipo aritmético a esta tarea se procede como sigue:

Solución (problema 1, nivel 0 de algebrización). Dado que tanto Juan como María usan el cuádruple de cucharadas de concentrado de jugo de limón que de cucharadas de azúcar, las dos limonadas tienen el mismo gusto.

Esta justificación se apoya en el conocimiento de las operaciones aritméticas (multiplicación y división de cantidades de magnitud) y se admite que ambas limonadas tendrán el mismo sabor si se mantiene la razón entre las cucharadas de azúcar y las cucharadas de jugo de limón. En la solución sólo intervienen valores numéricos de 
medidas y sus unidades, y se aplican operaciones aritméticas sobre dichos valores; por tanto, según Godino et al. (2014), la actividad matemática realizada se considera de nivel 0 de algebrización, en tanto que no intervienen objetos y procesos algebraicos.

\subsection{Del significado aritmético al protoalgebraico: Reducción a la unidad}

Una posible solución al problema anterior de la limonada podría haber pasado por determinar el número de cucharadas de concentrado de jugo de limón que usan tanto Juan como María por cada cucharada de azúcar:

Solución (problema 1, nivel 1 de algebrización). Como Juan emplea 12:3=4 cucharadas de concentrado por cada cucharada de azúcar y María utiliza 20:5=4 cucharadas de concentrado por cada cucharada de azúcar, resulta que, la razón de concentrado de limón por cucharada de azúcar es la misma en ambos casos, de modo que las dos limonadas estarán igual de dulce.

En una solución como la anterior, intervienen los siguientes objetos:

- Conceptos: cantidades de magnitud, razón unitaria, igualdad de razones.

- Procedimientos: reducción a la unidad.

- Proposiciones: las razones de concentrado de limón por cucharada de azúcar son iguales.

- Argumentos: si las razones unitarias coinciden en las limonadas el sabor es el mismo.

En un problema de valor faltante, la técnica de reducción a la unidad consiste en determinar la razón unitaria para luego multiplicar por el factor dado y obtener el resultado solicitado. El reconocimiento del valor unitario supone determinar una regla general compatible con una secuencia finita de cantidades de magnitudes directamente proporcionales, que permita generar los términos de la secuencia. Según los criterios de Godino et al. (2014), la actividad matemática se considera protoalgebraica de nivel 1. Pensemos en la siguiente situación-problema propia de Educación Primaria:

Problema 2. Es el cumpleaños de Lucía y quiere hacer una tarta de chocolate. En la receta que ha encontrado hay los ingredientes para 4 personas.

a) ¿Qué cantidad de galletas necesita para 6 personas? ¿Podrías determinar la cantidad de galletas para un número dado de comensales?
Tarta de chocolate (4 personas)

-24 galletas -6 huevos

$-100 \mathrm{~g}$ mantequilla $-300 \mathrm{~g}$ chocolate

b) ¿Qué cantidad de huevos necesita para 6 personas?

Figura 2. Ejemplo de problema con significado protoalgebraico

Se asume que para elaborar una tarta con la misma receta, la cantidad de ingredientes debe ser proporcional a la cantidad de personas. Si el número de comensales aumenta, aumentará la cantidad de cada ingrediente, y si se duplica, triplica, etc., el número de comensales, se duplicará, triplicará, etc., la cantidad de cada ingrediente. Así:

$$
\begin{aligned}
\text { Comensales } & \text { Galletas } \\
4 & \rightarrow 24 \\
1 & \rightarrow 24 / 4=6 \\
6 & \rightarrow 6 \times 6=36 \\
n & \rightarrow 6 \times n
\end{aligned}
$$

Solución (problema $2 a$, nivel 1 de algebrización). Si para 4 comensales se necesitan 24 galletas, por cada comensal, se necesitan $24: 4=6$ galletas. Para 6 comensales, necesitaremos $6 \times 6=36$ galletas, y para un número dado, $n$, de comensales se debe multiplicar $n$ por 6 (valor unitario) para determinar la cantidad necesaria de galletas.

El método de reducción a la unidad puede ser inadecuado en situaciones en las cuales las unidades no son fraccionables, llevando a un discurso poco claro o absurdo (Comin, 2000). Así, determinar la cantidad de huevos para preparar la tarta para 6 comensales, por medio de reducción a la unidad puede ser confuso, dado que para un comensal sería preciso $6 / 4=1,5$ huevos. Alternativamente, se puede seguir un procedimiento aritmético: 
Comensales Huevos

$4 \rightarrow 6$

$2 \rightarrow 6: 2=3$

$4+2=6 \rightarrow 6+3=9$
Solución (problema 2b, nivel 0 de algebrización). Si para 4 comensales necesito 6 huevos, para la mitad de comensales, es decir, para 2 comensales, necesitaré la mitad de huevos, esto es 3 , y para $4+2=6$ comensales, precisaré, $6+3=9$ huevos.

\subsection{Significado protoalgebraico: Proporciones, ecuación proporcional y secuencias de números proporcionales}

Si dos magnitudes A y B son directamente proporcionales, dos pares de valores numéricos de medidas de las magnitudes, determinan fracciones equivalentes. En tal caso, los productos cruzados de numeradores y denominadores serán iguales entre sí.

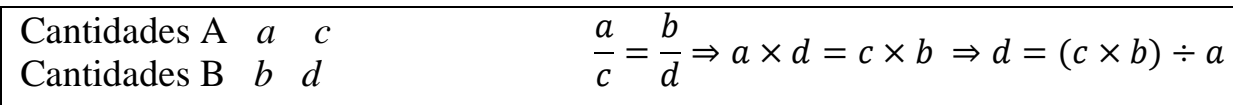

Una solución basada en la multiplicación en cruz de términos de las fracciones equivalentes determinadas por valores de magnitudes directamente proporcionales supone una actividad matemática de carácter protoalgebraico de nivel 1, en tanto aparecen objetos intensivos de segundo grado de generalidad, propiedades de la estructura algebraica de $\mathbb{N}$ y la igualdad como equivalencia. Tal sería el caso de la siguiente secuencia de prácticas, para determinar el número de galletas y de huevos necesarios para elaborar una tarta para 6 personas:

Solución (problema 2, nivel 1 de algebrización). Como la cantidad de galletas y de huevos es directamente proporcional a la cantidad de comensales, se tiene que:

Si para 4 comensales se necesitan 24 galletas, para 6 comensales, se necesitan $(24 \times 6) \div 4=36$ galletas // Si para 4 comensales se necesitan 6 huevos, para 6 comensales, se necesitan $(6 \times 6) \div$ $4=9$ huevos.

Los objetos que interviene en esta solución serían los siguientes:

- Conceptos: magnitud, cantidad, proporcionalidad directa, fracciones equivalentes.

- Procedimientos: aritméticos (multiplicación y división de términos en fracciones equivalentes).

- Proposiciones: si dos fracciones son equivalentes, su producto cruzado coincide.

- Argumentos: si dos magnitudes son directamente proporcionales, dos pares de valores numéricos de medidas de las magnitudes determinan fracciones equivalentes

\subsubsection{Ecuación proporcional y regla de tres}

El significado protoalgebraico está centrado en la aplicación de las nociones de razón y proporción. Según Freudenthal (1983), una razón es una función de un par ordenado (antecedente y consecuente) de números o de valores de una magnitud. El significado de razón reside en la posibilidad de comparar dos razones, es decir, afirmar con sentido que " $a$ es a $c$ " $(a: c)$ como " $b$ es a $d$ " $(b: d)$. El concepto de proporción se construye entonces sobre el de razón. Una proporción es una relación de igualdad entre dos razones. Cuando las razones se expresan como fracciones, la proporción " $a$ es a $c$ como $b$ es a $d$ ", escrita simbólicamente de la forma $a: c:: b: d$, aparece como igualdad de fracciones:

$$
\frac{a}{c}=\frac{b}{d}
$$

Cualquier cambio de disposición entre los cuatro números que forman una proporción que no modifique los productos cruzados de los numeradores y denominadores entre sí dará lugar a una nueva igualdad de fracciones. Así pues, 


$$
\frac{a}{c}=\frac{b}{d} \Leftrightarrow \frac{a}{b}=\frac{c}{d}
$$

Esto es, la permutación de los extremos o de los medios permite obtener una igualdad de razones externas (sus elementos pertenecen a espacios de medida distintos) a partir de la igualdad de razones internas (antecedente y consecuente comparten el mismo espacio de medida) o inversamente.

En un problema de valor faltante, si $a$ es a $c$ como $b$ es a $x$, siendo $a, b$ y $c$ números reales conocidos y $x$ el valor faltante, se trata de determinar este valor. Con las razones en forma de fracción, la proporción es manipulable como equivalencia de dos fracciones mediante la propiedad del producto en cruz para resolver la ecuación proporcional:

$$
\frac{a}{c}=\frac{b}{x} \Rightarrow a x=b c \Rightarrow x=\frac{b c}{a}
$$

La solución de un problema de valor faltante basada en el uso de proporciones, involucra una incógnita y el planteamiento de una ecuación en la que la incógnita está despejada en un miembro de la ecuación que se establece mediante la proporción. Por tanto, según el modelo de Godino et al. (2014), la actividad de algebrización que se realiza es de nivel 2 (protoalgebraica).

La regla de tres no es más que una variante diagramática de esta técnica que "oculta" la intervención de las razones y la proporción, lo que conlleva un significado "degenerado" de la proporcionalidad aritmética, esto es, reducido a la aplicación de rutinas algorítmicas sin justificación pertinente. En este caso consiste en la ordenación de los datos, generalmente en forma de representación tabular (también mediante flechas que expresan las razones). Por simple disposición de la tabla se infiere la ecuación proporcional, de la que se obtiene la cantidad buscada por manipulación algorítmica.

En el ejemplo anterior, existe una correspondencia de proporcionalidad directa entre las magnitudes "número de personas" y "número de galletas". Por tanto:

Solución (problema 2a, nivel 2 de algebrización). La razón de cantidades que se corresponden se mantiene constante, es decir, 4 personas: 24 galletas $:: 6$ personas: $x$ galletas, donde $x$ representa el valor de galletas para 6 personas. Establecida la proporción entre los valores de las magnitudes, $\frac{4}{24}=\frac{6}{x}$, y teniendo en cuenta la igualdad del producto en cruz de los términos en una proporción, $4 \times x=24 \times 6$, finalmente $x=\frac{24 \times 6}{4}=36$, por lo que se precisan 36 galletas para la receta.

En una solución como la anterior, basada en el establecimiento de la ecuación proporcional, intervienen los siguientes objetos de índole algebraica:

- Conceptos: razón de cantidades de magnitudes, proporción, incógnita, ecuación.

- Proposiciones: las razones de cantidades que se corresponden entre magnitudes proporcionales son iguales.

- Procedimientos: despeje de la incógnita en una ecuación del tipo $A x=B$.

- Argumentos: recaen en la relación de proporcionalidad directa y en las propiedades de razones y proporciones.

\subsubsection{Secuencias de números proporcionales}

Dos secuencias de números, que se corresponden uno a uno son proporcionales si las razones de los números correspondientes son iguales (equivalentemente existe un número real fijo $k$, que permite escribir cada valor de la segunda serie como producto de $k$ por los valores de la primera serie). Así pues, si entre dos magnitudes A y B existe una relación 
de proporcionalidad directa, las secuencias de los valores de las cantidades de ambas magnitudes determinan secuencias de números proporcionales.

Las secuencias de números proporcionales, y su representación mediante las habituales tablas de proporcionalidad, establecen un puente entre el significado aritmético o protoalgebraico centrado en la idea de proporción, y el propiamente algebraico centrado en la función lineal. El uso de tablas de proporcionalidad (Tabla 1) permite al alumno formular hipótesis y extraer conclusiones sobre los valores, variaciones y relaciones posibles entre los números de la tabla. Por ejemplo, permite reconocer una propiedad fundamental de las proporciones, a saber, que la suma de antecedentes dividida por la suma de los consecuentes de una proporción es igual a cualquiera de las razones de esa proporción, que a menudo se usa en problemas de repartos proporcionales. Así mismo, permite incorporar razones internas y externas, e introducir las propiedades de la función lineal (carácter aditivo y homogéneo) que consideraremos en la siguiente sección.

Tabla 1. Tabla de proporcionalidad

\begin{tabular}{|l|l|l|l|l|l|l|l|l|l|l|l|l|l|}
\hline $\begin{array}{l}\text { Cantidades de } \\
\text { magnitud } A\end{array}$ & $a_{1}$ & $a_{2}$ & $\ldots$ & $a_{i}$ & $\ldots$ & $a_{j}$ & $\ldots$ & $a_{i} a_{j}$ & $\ldots$ & $a_{k}$ & $\ldots$ & $n \times a_{k}$ & $\ldots$ \\
\hline $\begin{array}{l}\text { Cantidades de } \\
\text { magnitud } B\end{array}$ & $b_{1}$ & $b_{2}$ & $\ldots$ & $b_{i}$ & $\ldots$ & $b_{j}$ & $\ldots$ & $b_{i}+b_{j}$ & $\ldots$ & $b_{k}$ & $\ldots$ & $n \times b_{k}$ & $\ldots$ \\
\hline
\end{tabular}

\subsection{Significado algebraico-funcional: La función lineal}

Como afirman Comin (2000) y Bolea, Bosch y Gascón (2001), en el paso del discurso aritmético al discurso algebraico, la relación de proporcionalidad entre magnitudes se convierte en una relación de proporcionalidad entre variables numéricas (desprovistas de unidades y que representan valores de medidas de cantidades de magnitud). Asimismo, la relación de proporcionalidad en una tabla como la anterior se resume en una fórmula estandarizada $y=k x$, donde $k$ es el valor unitario o constante de proporcionalidad.

El significado propiamente algebraico se caracteriza por la aplicación de la noción de función lineal y de técnicas de resolución basadas en las propiedades de dicha función. A través de ella, la proporcionalidad se reconoce como una situación en la que existe una relación funcional multiplicativa constante entre dos magnitudes que covarían.

Una función lineal es una función real de variable real $f: \mathbb{R} \rightarrow \mathbb{R}$ donde la relación queda definida por $f(x)=k x$, con $k$ la constante de proporcionalidad (número real fijo).

Incluimos a continuación las propiedades fundamentales de la función lineal. Estas deben respaldar los argumentos empleados para justificar que una situación-problema dada es de proporcionalidad directa y asegurar la pertinencia de los procedimientos utilizados en la resolución de la misma.

A. Una función lineal $f: \mathbb{R} \rightarrow \mathbb{R}$ es homogénea, esto es, $f(\lambda x)=\lambda f(x), y$ aditiva, $f\left(x_{1}+x_{2}\right)=f\left(x_{1}\right)+f\left(x_{2}\right)$, para cualesquiera, $x, x_{1}, x_{2}$ en el dominio de definición de f $y \lambda \in \mathbb{R}$. Estas propiedades caracterizan a una función real de variable real como lineal y establecen las condiciones para definirla en estructuras algebraicas más generales como son las de espacios vectoriales.

B. Para una función $f: \mathbb{R} \rightarrow \mathbb{R}$ son equivalentes las afirmaciones siguientes:

i) $f$ es monótona y aditiva

ii) $f$ es monótona $y \mathbb{N}$-lineal, esto es, $f(n x)=n f(x)$, para todo $n \in \mathbb{N}$ y todo $x$ en el dominio de definición de $f$ 


\section{iii) $f$ es lineal}

Las afirmaciones $i$ ) y $i$ i) se relacionan con las formas intuitivas de enunciar la proporcionalidad: "a más...más" o "a menos...menos" (monotonía), "si una magnitud se duplica, triplica, etc. entonces la magnitud correspondiente también se duplica, triplica, etc. $(\mathbb{N}$-linealidad).

C. Si una función $f: \mathbb{R} \rightarrow \mathbb{R}$ es $\mathbb{N}$-lineal y continua, entonces $f$ es lineal. La continuidad, al igual que ocurre con la monotonía, son condiciones implícitas de forma habitual en el contexto que se modeliza por medio de la función lineal.

\section{Si $f: \mathbb{R}_{0}^{+} \rightarrow \mathbb{R}_{0}^{+}$es aditiva entonces $f$ es creciente $y f(x)=f(1) x$}

La solución a un problema basada en el uso de la noción de la función lineal y de las propiedades descritas, supone según Godino et al. (2014) un nivel 3 de algebrización ya que la representación de la función lineal en lenguaje formal requiere el uso de lenguaje simbólico-literal y que se opere de manera analítica/sintáctica con dicho lenguaje. Pensemos en la siguiente situación-problema y una posible solución:

Problema 3. $10 \mathrm{dm}^{3}$ de madera de abeto pesan 4,5kg. Determina la expresión analítica de la función que relaciona la masa y el volumen de la madera de abeto. ¿Cuál es la densidad de este material?

Solución (nivel 3 de algebrización). El peso de distintos trozos de un mismo material es directamente proporcional a su volumen. Es decir, el peso es mayor a medida que es mayor el volumen y el peso de un trozo de volumen igual a la suma de los volúmenes de dos trozos por separado, es igual a la suma de los pesos de dichos trozos menores. Teniendo esto en cuenta, la correspondencia $f: \mathbb{R}^{+} \rightarrow \mathbb{R}^{+}$que se establece entre el volumen de distintas piezas de madera de abeto y el peso de las mismas es creciente y aditiva. Por tanto, $f$ es lineal. Es decir, $f(x)=k x$, donde $k=f(1)$ es la constante de proporcionalidad y representa la densidad de la madera de abeto (es decir, la masa por unidad de volumen).

Puesto que $10 \mathrm{dm}^{3}$ de madera de abeto pesan $4,5 \mathrm{~kg}$ sabemos que $f(10)=4,5$. Aplicando las propiedades de la función lineal, $10 f(1)=4,5 ; f(1)=4,5 \div 10=0,450$. Por tanto, la función que determina la masa (en kilogramos) de un trozo con un determinado volumen (en $\mathrm{dm}^{3}$ ) de madera de abeto es $f(x)=0,450 x$ y la densidad de la madera de abeto es 0,450 $\mathrm{kg} / \mathrm{dm}^{3}$, o lo que es lo mismo $450 \mathrm{~g} / \mathrm{m}^{3}$.

Una solución como la anterior, basada en el reconocimiento de las características que definen la función lineal incorpora nuevos objetos que corresponden a un nivel consolidado de algebrización:

- Conceptos: correspondencia funcional, variable, dominio e imagen de una aplicación, función lineal, coeficiente de proporcionalidad.

- Proposiciones: una función $f: \mathbb{R}^{+} \rightarrow \mathbb{R}^{+}$aditiva y creciente es lineal.

- Procedimientos: traducción del lenguaje natural al simbólico.

- Argumentos: recaen en las propiedades que caracterizan una función lineal y el significado de la constante de proporcionalidad.

Como señala García (2005) la sustitución de la teoría de razones y proporciones por la teoría de funciones reales de variable real, supone la inmersión de la proporcionalidad en el universo de las relaciones funcionales entre magnitudes. Una de estas posibles relaciones es la que García refiere como relación de variación equitativa. La relación entre dos magnitudes $\mathrm{M}$ y M' es de variación equitativa si a toda progresión aritmética de valores de cantidades de la magnitud $\mathrm{M}$, corresponde una progresión aritmética de valores de cantidades de magnitud M' (García, 2005, p. 203). El modelo funcional de dicha condición de equitatividad es la función afín. Desde el punto de vista de la teoría de 
funciones reales de variable real, la función afín $f(x)=m x+n$, puede obtenerse como una traslación de la función lineal, $g(x)=m x$. En particular, una función lineal $g: \mathbb{R} \rightarrow$ $\mathbb{R}$ es un caso de función afín tal que $g(0)=0$.

\subsection{Familias de funciones lineales. Operaciones con funciones lineales}

Bajo el modelo matemático $y=k \cdot x$ se pone en juego el conocimiento de la estructura de una familia de funciones, $\left\{f_{k}: \mathbb{R} \rightarrow \mathbb{R} / f_{k}(x)=k x ; k \in \mathbb{R}\right\}$, puesto que $k$ interviene como parámetro, lo cual es un primer contacto con el nivel 4 de algebrización en Godino et al. (2015). El estudio de las operaciones con funciones y de sus propiedades estructurales implica un nivel superior de algebrización (nivel 5). Volvamos a la situación-problema que analizamos en la sección previa, incorporando nuevas relaciones:

Problema 4. Si se mezclan virutas de abeto y de roble a razón $2: 3$, ¿cuál es la densidad de la mezcla?

Solución (nivel 5 de algebrización). La densidad de la mezcla de madera expresa la relación entre la masa resultante de la mezcla de los distintos tipos de maderas viruteadas después del proceso de molido y el volumen que ocupan. Sean $f_{a}(x)=k_{a} x, f_{r}(x)=k_{r} x$, y $f_{m}(x)=$ $k_{m} x$, respectivamente, las funciones que expresan la masa de un trozo de madera de abeto, roble y de mezcla, a partir del volumen del mismo. Si de un trozo de madera de mezcla de volumen $x$, un volumen $u$ es de abeto y un volumen $v$ es de roble:

$$
f_{m}(x)=f_{m}(u+v)=f_{a}(u)+f_{r}(v)=k_{a} u+k_{r} v
$$

Dado que se mezclan virutas de abeto y de roble a razón 2:3, $u=\frac{2}{5} x$ y $v=\frac{3}{5} x$ de manera que $f_{m}(x)=k_{a} \frac{2}{5} x+k_{r} \frac{3}{5} x=\left(\frac{2}{5} k_{a}+\frac{3}{5} k_{r}\right) x$, luego la densidad de la mezcla es $k_{m}=$ $f_{m}(1)=\left(\frac{2}{5} k_{a}+\frac{3}{5} k_{r}\right)$

Identificamos los siguientes objetos en la solución propuesta:

- Conceptos: masa, volumen, densidad, razón, función lineal, variable, parámetro.

- Proposiciones: La densidad de la mezcla de madera expresa la relación entre la masa resultante de la mezcla de los distintos tipos de maderas viruteadas después del proceso de molido y el volumen que ocupan

- Procedimientos: traducción del lenguaje natural al lenguaje simbólico, operaciones con funciones lineales y parámetros

- Argumentos: recaen en las propiedades de las familias de funciones lineales y en las operaciones entre éstas.

Los coeficientes de las funciones lineales que determinan la relación entre la masa y el volumen de ambos tipos de madera actúan cómo parámetros. Las operaciones con dichos parámetros, conjuntamente con variables y el establecimiento de relaciones entre ellos, implican una actividad algebraica de nivel 5 dado que los objetos que intervienen y emergen de estos sistemas de prácticas ponen en juego a los objetos algebraicos del nivel previo (familia de funciones lineales).

\subsection{Aplicaciones lineales y espacios de medida}

La introducción de estructuras algebraicas como las de espacio vectorial o espacio de medida, así como el estudio del álgebra de aplicaciones en dichas estructuras, ponen en juego objetos y procesos algebraicos de mayor grado de generalidad que los considerados en el quinto nivel, quedando reservados para estudios universitarios. 
Un espacio vectorial sobre un cuerpo $\mathbb{K}$ es una terna $(\mathrm{V},+, \cdot)$ formada por un conjunto $\mathrm{V}$ no vacío (cuyos elementos se llaman vectores) y dos operaciones, una de ellas interna, suma de vectores $+: V \times V \rightarrow V$, respecto de la que $\mathrm{V}$ es un grupo abeliano, y otra externa, el producto por escalares $:: \mathbb{K} \times V \rightarrow V$ que verifican propiedades compatibles con la estructura de grupo. Todo cuerpo es un espacio vectorial sobre sí mismo, de manera que la estructura de espacio vectorial generaliza la del cuerpo de los números reales.

Dados dos espacios vectorial $\mathrm{V}$ y $\mathrm{W}$ sobre un cuerpo $\mathbb{K}$, una aplicación $f: V \rightarrow W$ se dice que es lineal si satisface:

$$
\begin{aligned}
& \text { 1. } f(u+v)=f(u)+f(v) \\
& \text { 2. } f(\lambda u)=\lambda f(u)
\end{aligned}
$$

para cualesquiera $\lambda \in \mathbb{K}, u, v \in V$. Es decir, $f$ es un morfismo de grupos abelianos $(V,+),(W,+)$ y conmuta con el producto por escalares.

En el siguiente problema se plantea un primer encuentro con la estructura algebraica de espacio vectorial y de aplicación lineal entre espacios vectoriales. Se ponen en juego objetos matemáticos, vectores y aplicaciones entre espacios de vectores, sobre los cuales se definen operaciones que cumplen un sistema de propiedades específicas.

Problema 5. Sea $V$ un espacio vectorial real y $U, W$ dos subespacios vectoriales de $V$. Sea $f: U \times W \rightarrow V$ la aplicación definida por $f(u, w)=u+w$. Demuestra que $f$ es una aplicación lineal.

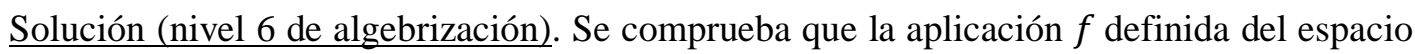
vectorial producto $U \times W$ en $V$ verifica las condiciones que definen la aplicación lineal.

$$
\begin{gathered}
f\left((u, w)+\left(u^{\prime}, w^{\prime}\right)\right)=f\left(u+u^{\prime}, w+w^{\prime}\right)=\left(u+u^{\prime}\right)+\left(w+w^{\prime}\right) \\
=(u+w)+\left(u^{\prime}+w^{\prime}\right)=f(u, v)+f\left(u^{\prime}, w^{\prime}\right) \\
f(\lambda(u, w))=f(\lambda u, \lambda w)=\lambda u+\lambda w=\lambda(u+w)=\lambda f(u, w)
\end{gathered}
$$

Una solución como la anterior, incorpora objetos que corresponden al nivel 6 de algebrización según el modelo de Godino et al. (2015):

- Conceptos: espacio vectorial, subespacio, producto cartesiano, aplicación lineal.

- Proposiciones: La suma de vectores es asociativa; se cumple la propiedad distributiva del producto por escalares respecto de la suma de vectores.

- Procedimientos: cálculo analítico (sintáctico) con vectores en un espacio vectorial y en el espacio vectorial producto.

- Argumentos: basados en la estructura de espacio del vectorial del producto cartesiano de dos espacios vectoriales y en las propiedades que definen la aplicación lineal.

Desde un punto de vista axiomático, una magnitud es un conjunto no vacío $M$, donde está definida una operación interna, la suma $+: M \times M \rightarrow M$, y una relación de orden total $<$, de manera que $(M,+,<)$ es un monoide, conmutativo, cancelativo y arquimediano. Fiol y Fortuny (1990) asumen además la propiedad de divisibilidad, según la cual, para todo $a$ en $M$ y $n$ entero positivo existe $b \in \mathrm{M}$, tal que $a=n b$. Para estos autores, los elementos de $M$ se llaman cantidades. Fijado un elemento unidad $e$ en $\mathrm{M}$, se define la medida del elemento $a$ con respecto a $e$, como el número real $\mu_{e}(a)=\sup \left\{q \in \mathbb{Q}^{+}: q e \leq a\right\}$. La magnitud $M$ se dice continua si la medida $\mu_{e}$ establece un isomorfismo entre $M$ y $\mathbb{R}_{0}^{+}$.

La proporcionalidad de dos magnitudes $M$ y $N$ viene dada por la existencia de un isomorfismo $f: M \rightarrow N$ que conserva el orden, esto es, para cualesquiera $a, b \in M$,

$$
\text { 1) } f(a+b)=f(a)+f(b)
$$


2) si $a<b$ entonces $f(a)<f(b)$

La propiedad 1) expresa el carácter aditivo de la aplicación, de manera que si $M=$ $N=\mathbb{R}$ con la suma y el orden habitual, la aplicación $f: M \rightarrow N$ es aditiva (luego $\mathbb{N}$-lineal) y la propiedad 2) se traduce en su crecimiento, es decir, $f$ es lineal.

Además, si dos magnitudes continuas $M$ y $N$ con unidades $e$ y $u$, son proporcionales con isomorfismo $f: M \rightarrow N$, la medida de $f(a)$ con unidad $f(e)$ será la misma que la medida de $a$ unidad $e$ y la aplicación $g: \mathbb{R}_{0}^{+} \rightarrow \mathbb{R}_{0}^{+}$, definida por $g(x)=\mu_{u}(f(x e))$, es aditiva. Por la propiedad D de las funciones lineales, $g(x)=g(1) x$, es la función lineal representante de la situación de proporcionalidad entre las magnitudes $M$ y $N$, y $k=g(1)$ es la constante de proporcionalidad.

\section{Síntesis y estructuración de los significados de la proporcionalidad}

La reconstrucción del significado de la proporcionalidad es el primer paso para comprender los procesos de enseñanza implementados y establecer pautas de mejora. Desde el punto de vista del EOS, los significados se interpretan en términos de subsistemas de prácticas institucionales ligadas a contextos de uso y de objetos emergentes (situaciones, lenguajes, conceptos, proposiciones, procedimientos y argumentos). Cada configuración epistémica y sus prácticas modelizan aspectos parciales del significado global del objeto proporcionalidad que debe ser el referente en una investigación de didáctica de la matemática centrada en el estudio de este objeto.

En la Figura 3 resumimos los significados de la proporcionalidad discutidos en el apartado anterior, haciendo referencia al nivel educativo en el que usualmente se aborda su estudio y el nivel de algebrización. A continuación reflexionamos sobre la presencia, tratamiento y problemática en torno a estos significados en la práctica educativa.

Entre los significados de proporcionalidad se establecen relaciones de interdependencia, pudiendo unos significados estar mejor adaptados a unas circunstancias y etapas educativas que otros. En la mayoría de los manuales escolares de primaria, se definen dos magnitudes como directamente proporcionales si al multiplicar o dividir una de ellas por un número, la otra queda multiplicada o dividida por el mismo número. Los argumentos para justificar si las magnitudes son directamente proporcionales también son incompletos y solo contemplan el carácter $\mathbb{N}$-lineal de la relación de proporcionalidad.

El método de reducción a la unidad, se introduce como primer procedimiento de resolución de problemas de proporcionalidad, apoyado en el conocimiento y sentido de las operaciones del alumno. El carácter algorítmico otorgado al tratamiento de la proporcionalidad en esta etapa, lleva a que los alumnos con frecuencia asocien la relación de proporcionalidad con la posibilidad de aplicar la regla de tres a la situación-problema, sin considerar las magnitudes involucradas ni los argumentos que sustentan la relación.

Las nociones de razón y proporción no se introducen hasta la Educación Secundaria, etapa en el que se espera que los alumnos tengan los conocimientos y práctica suficiente sobre fracciones que permita dotar de sentido al producto de magnitudes de naturaleza diferente. En esta etapa se introduce la constante (o razón) de proporcionalidad y la relación de proporcionalidad se define a partir de estas. El tratamiento es aritmético y previo al estudio de magnitudes y su medida. La relación de proporcionalidad de dos magnitudes se establece de manera implícita por la igualdad de razones entre sus medidas.

Las secuencias proporcionales y el registro tabular permiten avanzar en el tratamiento de la proporcionalidad hacia niveles superiores, conectando los significados aritmético 
(basado en relaciones aditivas y multiplicativas doble-mitad) y protoalgebraico (valor unitario, proporciones) con las propiedades de la función lineal. Las tablas de proporcionalidad recogen un número finito de parejas de valores correspondientes de cantidades de magnitudes proporcionales, y permiten hacer hipótesis sobre los valores, las variaciones y detectar relaciones posibles (relaciones entre razones internas y razones externas, propiedad aditiva, crecimiento, $\mathbb{N}$-linealidad) entre los números de la tabla.

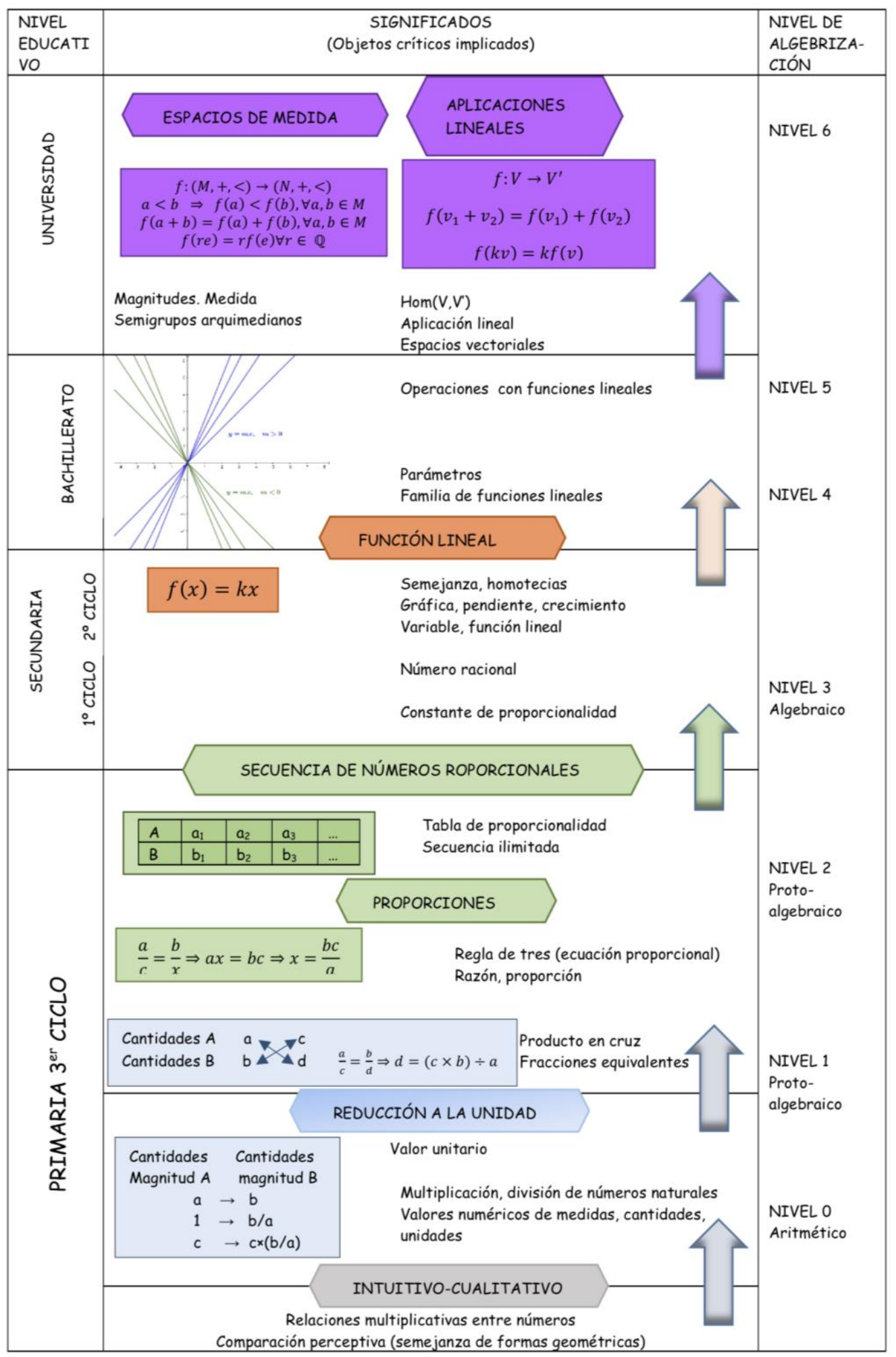

Figura 3. Significados de la proporcionalidad según niveles de algebrización y educativo 
En Educación Secundaria, la función lineal y la función afín son los primeros ejemplos de funciones reales de variable real. Éstas vienen asociadas a las tablas de valores y al registro gráfico pero contribuyen a hacer desaparecer las magnitudes de los modelos matemáticos al reflejar solo los valores numéricos de las cantidades que intervienen sin expresión de las unidades de medida correspondientes. Por otro lado, la función lineal $f(x)=k x$ introduce una asimetría en una relación, la de proporcionalidad, que es simétrica: la variable $x$ se diferencia de su imagen $\mathrm{y}=f(x)$. Como afirma Comin (2000, p. 127) "un trabajo reflexivo puede hacer aparecer la función lineal como modelización numérica de la relación de proporcionalidad entre magnitudes".

La inmersión de la relación de proporcionalidad en el universo de las funciones lineales, permite considerar la función lineal como un caso más de posible relación entre variables numéricas y recurrir a las técnicas que el contexto funcional facilita. La función lineal deja paso en niveles superiores de algebrización a las aplicaciones lineales entre espacios vectoriales, donde las propiedades características de las funciones lineales sirven de definición y aparece una nueva álgebra de aplicaciones entre espacios vectoriales, pero desaparece la idea de magnitud por la razón antes mencionada. Un modelo general de la proporcionalidad, requiere de la teoría de medida, donde las magnitudes adquieren el carácter más abstracto de monoides conmutativos, cancelativos, arquimedianos, y la proporcionalidad de magnitudes es un isomorfismo entre las estructuras.

\section{Reflexiones finales}

Diversas investigaciones señalan que, tanto los profesores en formación inicial como en activo de distintas etapas tienen una escasa comprensión de la proporcionalidad, basada en conocimientos procedimentales como el producto cruzado o regla de tres de los que desconocen su componente conceptual (Lamon, 2007; Riley, 2010). Consideramos que la visión global sobre el significado de la proporcionalidad elaborada en este trabajo puede ayudar a introducir cambios fundamentados en los programas de formación de profesores que permitan superar las limitaciones mencionadas.

El profesor de matemáticas de un nivel educativo debe conocer las matemáticas escolares de ese nivel, pero también debe poder articular esos conocimientos con los correspondientes a niveles posteriores (Godino, Giacomone, Batanero \& Font, 2017). Esto supone conocer los diversos significados de los objetos matemáticos, los informales y formales y sus interconexiones. La identificación del profesor de los objetos y procesos que intervienen en las prácticas matemáticas es una competencia que le permitirá comprender la progresión de aprendizajes, gestionar los procesos de institucionalización y evaluar las competencias matemáticas de los alumnos. Es claro que el profesor debe tener, además, conocimientos relacionados con las orientaciones curriculares, desarrollo cognitivo y conflictos de aprendizaje, así como ser competente en el diseño y gestión de recursos didácticos específicos para el estudio de la proporcionalidad (Ben-Chaim, Keret \& Ilany, 2012; Fiol \& Fortuny, 1990; Godino \& Batanero, 2002). La perspectiva ofrecida en este trabajo sobre los tipos de significados de la proporcionalidad ligados a los niveles de algebrización señalan una trayectoria para la planificación curricular que contemple el progresivo desarrollo de la competencia matemática de los estudiantes, la cual no debería estar limitada, para el caso de este contenido matemático, al aprendizaje de las nociones de razón, proporción y a la aplicación de rutinas estereotipadas.

El foco de atención de este trabajo ha sido identificar los distintos significados asociados al objeto proporcionalidad, y articularlos en un modelo ontosemiótico que pueda servir de referencia para el diseño instruccional. Los niveles de algebrización han 
permitido modelizar el conocimiento institucional que se pone en juego en las prácticas operativas, discursivas y normativas implicadas en la resolución de problemas de proporcionalidad, describiendo la actividad matemática bajo la perspectiva de objetos y procesos característicos del álgebra, admitiendo que un mismo problema se puede abordar de diferentes maneras en un momento dado con niveles de algebrización diferentes.

Como se indica en Godino et al. (2017), entre los diversos significados del objeto proporcionalidad se establecen relaciones de interdependencia, simbiosis y cooperación; pudiendo unos significados estar mejor adaptados en unas circunstancias que en otras. Los significados, geométrico, probabilístico, estadístico, o de otros contextos, requieren de la cooperación de los significados aritmético o proto-algebraico para dar respuesta a las cuestiones que involucran el razonamiento proporcional. La regla de tres, en su interpretación aritmética, o incluso en su versión algorítmica/instrumental tiene de hecho su nicho ecológico en el contexto de la vida cotidiana o incluso en los contextos técnicoprofesionales y artísticos. No obstante, dado el papel esencial del álgebra en las distintas ramas de la matemática, la implementación del significado algebraico de la proporcionalidad en la escuela secundaria es un factor positivo para el progreso del aprendizaje matemático de los estudiantes.

El reconocimiento de niveles de algebrización vinculados a los distintos significados que hemos precisado en la sección 3 constituye un aspecto importante de la faceta epistémica del conocimiento didáctico-matemático del profesor requerido para una enseñanza idónea de este contenido. Por otro lado, es también importante que los profesores conozcan de manera explícita las propiedades que definen la relación de proporcionalidad, a través de la función lineal, así como los argumentos que, sustentados en dichas propiedades, justifican la presencia o ausencia de proporcionalidad y la pertinencia de técnicas y procedimientos en determinadas situaciones. Como afirman Wilhelmi, Godino y Lacasta (2014, p. 581) “el desempeño como profesores se puede ver seriamente perjudicado si no se complementa con una profundización en la formación epistemológica específica sobre la pluralidad de significados de los objetos matemáticos y las configuraciones de objetos y procesos en los cuales cristalizan tales significados".

\section{Agradecimientos}

EDU2016-74848-P (FEDER, AEI); FQM-126 (Junta de Andalucía, España).

\section{Referencias}

Batanero, C. (2005). Significados de la probabilidad en la educación secundaria. RELIME, 8(3), 247-264.

Ben-Chaim, D., Keret, Y. y Ilany, B. S. (2012). Ratio and proportion: Research and teaching in mathematics teachers' education. Roterdam: Sense Publishers.

Behr, M., Harel, G., Post, T. y Lesh, R. (1992). Rational number, ratio and proportion. En D. A. Grouws (Ed.), Handbook of research on mathematics teaching and learning (pp. 296-333). Nueva York: Macmillan.

Bolea, P. (2002). El proceso de algebrización de organizaciones matemáticas escolares. Trabajo de Tesis Doctoral. Universidad de Zaragoza.

Bolea, P., Bosch, M. y Gascón, J. (2001). La transposición didáctica de organizaciones matemáticas en procesos de algebrización: El caso de la proporcionalidad. Recherches en Didactique des Mathématiques, 21(3), 247-304. 
Bosch M. (1994). La dimensión ostensiva en la actividad matemática. El caso de la proporcionalidad. Trabajo de Tesis Doctoral. Universitat Autònoma de Barcelona.

Chevallard, Y. (1992). Concepts fondamentaux de la didactique: Perspectives apportées par une approche anthropologique. Recherches en Didactique des Mathématiques, 12 (1), 73-112.

Comin, E. (2000). Proportionnalité et fonction linéaire Caractères, causes et effets didactiques des évolutions et des réformes dans la scolarité obligatoire. Histoire et perspectives sur les mathématiques [math.HO]. Université Sciences et Technologies Bordeaux I.

Cramer, K. y Post, T. (1993). Connecting research to teaching proportional reasoning. Mathematics Teacher, 86(5), 404-407.

Fernández, C. y Llinares, S. (2012) Características del desarrollo del razonamiento proporcional en la Educación Primaria y Secundaria. Enseñanza de las Ciencias, 30(1), 129-142.

Fiol, M. L. y Fortuny, J. M. (1990). Proporcionalidad directa. La forma y el número. Madrid: Síntesis.

Freudenthal, H. (1983). Didactical phenomenology of mathematical structures. Hingham, MA: Kluwer Academic Publishers.

García F. J. (2005). La modelización como herramienta de articulación de la matemática escolar. De la proporcionalidad a las relaciones funcionales. Trabajo de Tesis Doctoral. Universidad de Jaén.

Godino, J. D. (2002). Un enfoque ontológico y semiótico de la cognición matemática. Recherches en Didactiques des Mathematiques, 22 (2), 237-284.

Godino, J. D., Aké, L., Gonzato, M. y Wilhelmi, M. R. (2014). Niveles de algebrización de la actividad matemática escolar. Implicaciones para la formación de maestros. Enseñanza de las Ciencias, 32(1), 199-219.

Godino, J. D. y Batanero, C. (1994). Significado institucional y personal de los objetos matemáticos. Recherches en Didactique des Mathématiques, 14(3), 325-355.

Godino, J. D. y Batanero, C. (2002). Proporcionalidad y su didáctica para maestros. Departamento de Didáctica de las Matemáticas. Universidad de Granada.

Godino, J. D. Batanero, C. y Font, V. (2007). The onto-semiotic approach to research in mathematics education. ZDM, 39 (1-2), 127-135.

Godino, J. D., Beltrán-Pellicer, P., Burgos, M. y Giacomone, B. (2017). Significados pragmáticos y configuraciones ontosemióticas en el estudio de la proporcionalidad. En J. M. Contreras et al. (Eds.), Actas del II Congreso International Virtual sobre el Enfoque Ontosemiótico del Conocimiento y la Instrucción Matemáticos. Disponible en http://enfoqueontosemiotico.ugr.es/civeos.html

Godino, J. D., Font, V., Wilhelmi, M. R. y Lurduy, O. (2011). Why is the learning of elementary arithmetic concepts difficult? Semiotic tools for understanding the nature of mathematical objects. Educational Studies in Mathematics, 77(2), 247-265.

Godino, J. D., Giacomone, B., Batanero, C. y Font, V. (2017). Enfoque ontosemiótico de los conocimientos y competencias del profesor de matemáticas. Bolema, 31(57), 90113. 
Godino, J. D., Neto, T., Wilhelmi, M. R., Aké, L., Etchegaray, S. y Lasa, A. (2015). Niveles de algebrización de las prácticas matemáticas escolares. Articulación de las perspectivas ontosemiótica y antropológica. Avances de Investigación en Educación Matemática, 8, 117-142.

Gómez, P. (2007). Desarrollo del conocimiento didáctico en un plan de formación inicial de profesores de matemáticas de secundaria. Trabajo de Tesis Doctoral. Universidad de Granada.

Lamon, S. (2007). Rational number and proportional reasoning. Toward a theoretical framework for research. En F. K. Lester (Ed.), Second handbook of research on mathematics teaching and learning (Vol. 1, pp. 629-667). Nueva York: IAP.

Lamon, S. (2012). Teaching fractions and ratios for understanding: Essential content knowledge and instructional strategies for teachers. Nueva York: Taylor \& Francis.

Obando, G., Vasco, C. E. y Arboleda, L. C. (2014). Enseñanza y aprendizaje de la razón, la proporción y la proporcionalidad: Un estado del arte. RELIME, 17(1), 59-81.

Pino-Fan, L., Godino, J. D. y Font, V. (2011). Faceta epistémica del conocimiento didáctico-matemático sobre la derivada. Educação Matemática Pesquisa, 13(1), 141178.

Riley, K (2010) Teachers' understanding of proportional reasoning. En P. Brosnan, D. B. Erchick y L. Flevares (Eds), Proceedings 32 ${ }^{\text {nd }}$ PME-NA (Vol. 6, pp. 1055-1061) Columbus, $\mathrm{OH}$ : PME-NA.

Ruiz, E. y Lupiáñez, J. L. (2009). Detección de obstáculos psicopedagógicos en la enseñanza y el aprendizaje de los tópicos de razón y proporción en alumnos de sexto grado de Educación Primaria. Electronic Journal of Research in Educational Psychology, 7(1), 397-424.

Silvestre, A. I. y Ponte, J. P. (2011) Una experiencia de enseñanza dirigida al desarrollo del razonamiento proporcional. Revista Educación y Pedagogía, 23(59), 137-158.

Steinbring, H. (1997). Epistemological investigation of classroom interaction in elementary mathematics teaching. Educational Studies in Mathematics 32, 49-92.

Streefland, L. (1985). Search for roots of ratio: Some thoughts on the long term learning process. Educational Studies in Mathematics, 16, 75-94.

Tourniaire, F. y Pulos, S. (1985). Proportional reasoning: A review of the literature. Educational Studies in Mathematics, 16, 181-204.

Wilhelmi, M. R. (2017). Proporcionalidad en Educación Primaria y Secundaria. En J. M. Contreras y otros (Eds.), Actas del II Congreso International Virtual sobre el Enfoque Ontosemiótico del Conocimiento y la Instrucción Matemáticos. Disponible en enfoqueontosemiotico.ugr.es/civeos.html

Wilhelmi, M. R., Godino, J. D. y Lacasta, E. (2007). Configuraciones epistémicas asociadas a la noción de igualdad de números reales. Recherches en Didactique des Mathematiques, 27 (1), 77-120.

\section{Referencias de los autores}

María Burgos, Universidad de Granada. mariaburgos@ugr.es

Juan D. Godino, Universidad de Granada.jgodino@ugr.es 


\title{
Ontosemiotic reference model of proportionality: Implications for curricular design in primary and secondary education
}

\author{
María Burgos, Universidad de Granada. mariaburgos@ugr.es \\ Juan D. Godino, Universidad de Granada.jgodino@ugr.es
}

The diversity of meanings and their progressive articulation, as well as the different degrees of generality and formalization should be considered in the curricular design of a mathematical content at the different educational levels. In this paper, we analyze the different meanings of proportionality, by using the Ontosemiotic Approach theoretical tools and the model of mathematical activity algebrization levels. We interpret meaning in terms of systems of operative and discursive practices related to solving problems. Taking into account prior research, we identify types of situations-problems and configurations (of practices, objects and processes), which are used to describe the partial meanings that must be considered in teaching and learning proportionality in primary and secondary education. The holistic meaning of proportionality includes the following partial meanings: 1) Intuitive-qualitative; 2) Reduction to unity; 3) Proportions; 4) Sequences of proportional numbers; 5) Linear function; 6) Linear applications; 7) Measurable spaces. We identify the specific situations linked to each partial meaning, as well as the critical mathematical objects (languages, concepts, procedures, propositions) put at stake, which characterize the corresponding level of algebrization. The intuitivequalitative meaning, reduction to unity and use of proportions can be introduced at the third cycle of primary education; the sequences of proportional numbers and linear function in compulsory secondary education and high school, while the meanings that involve linear applications and measurable spaces are typical of university levels. Being able to recognize the algebrization levels linked to the different partial meanings (epistemic facet) is an important aspect of the teacher didactic-mathematical knowledge required for a suitable teaching of proportionality. Moreover, teachers should explicitly know the properties that define the proportionality relationship, through the linear function, the arguments that justify the presence or absence of proportionality, and the relevance of applying the corresponding techniques and procedures. Several studies indicate that, both prospective and in service teachers across educational levels have a poor understanding of proportionality, usually based on procedural knowledge, as well as on the cross product or rule of three, with lack of conceptual components. The global vision on the meaning of proportionality presented in this paper can serve to improve teacher education programs in order to overcome the mentioned limitations. 